\title{
Tuberculose e silicose: epidemiologia, diagnóstico e quimioprofilaxia*
}

\author{
Tuberculosis and silicosis: epidemiology, diagnosis and chemoprophylaxis \\ Carlos Eduardo Galvão Barboza', Daniel Hugo Winter², Márcia Seiscento ${ }^{3}$, \\ Ubiratan de Paula Santos ${ }^{4}$, Mário Terra Filho ${ }^{5}$
}

\begin{abstract}
Resumo
A silicose, a mais prevalente das pneumoconioses, é provocada pela inalação de partículas de sílica cristalina. Indivíduos expostos à sílica, com ou sem silicose, apresentam risco aumentado de tuberculose e de micobacterioses não-tuberculosas. 0 risco de silicóticos desenvolverem tuberculose em relação a controles sadios varia de 2,8 a 39 vezes, em conformidade com a gravidade da doença de base. Têm sido estudados diferentes esquemas de quimioprofilaxia para tuberculose em silicóticos, todos com eficácia semelhante e com redução final de risco para cerca da metade em relação ao uso de placebo. São, no entanto, esquemas de longa duração, o que, acrescido dos possíveis efeitos colaterais (particularmente hepatotoxicidade), podem prejudicar a aderência. As diretrizes atuais recomendam a realização de prova tuberculínica e, se positiva, a instituição de quimioprofilaxia. São vários os esquemas possíveis, tanto em termos de drogas quanto de duração. Nossa recomendação é de que se use isoniazida na dose de $300 \mathrm{mg} /$ dia (ou $10 \mathrm{mg} / \mathrm{kg} /$ dia) por seis meses para os indivíduos com silicose ou sadios com exposição superior a 10 anos, se forem reatores fortes à prova tuberculínica (induração $\geq 10$ mm). São necessários, no entanto, novos estudos para que indicações, drogas, doses e duração da profilaxia sejam definidas mais apropriadamente.
\end{abstract}

Descritores: Silicose; Tuberculose; Técnicas de diagnóstico e procedimentos; Quimioprevenção.

\begin{abstract}
Silicosis, the most prevalent of the pneumoconioses, is caused by inhalation of crystalline silica particles. Silica-exposed workers, with or without silicosis, are at increased risk for tuberculosis and nontuberculous mycobacteria-related diseases. The risk of a patient with silicosis developing tuberculosis is higher (2.8 to 39 times higher, depending on the severity of the silicosis) than that found for healthy controls. Various regimens for tuberculosis chemoprophylaxis in patients with silicosis have been studied, all of which present similar efficacy and overall risk reduction to about one half of that obtained with placebo. long-term regimens have potential side effects (particularly hepatotoxicity). In addition, the use of such regimens can jeopardize adherence to treatment. The current guidelines recommend that tuberculin skin tests be performed, and, if positive, that chemoprophylaxis be instituted. There are several possible regimens, varying in terms of the drugs prescribed, as well as in terms of treatment duration. We recommend the use of isoniazid at $300 \mathrm{mg} / \mathrm{day}$ (or $10 \mathrm{mg} / \mathrm{kg} / \mathrm{day}$ ) for six months for patients with silicosis, as well as for healthy patients with periods of exposure to silica longer than 10 years and strongly positive tuberculin skin test results (induration $\geq 10 \mathrm{~mm}$ ). Nevertheless, further studies are necessary so that indications, drugs, doses and duration of chemoprophylaxis regimens can be more properly defined.
\end{abstract}

Keywords: Silicosis; Tuberculosis; Diagnostic techniques and procedures; Chemoprevention.

\section{lntrodução}

A silicose é uma doença pulmonar fibrosante provocada pela inalação e deposição de partículas de sílica cristalina causando uma reação do pulmão. Dentre as pneumoco-

nioses, é a mais prevalente. São ambientes com maior risco ocupacional: extração e beneficiamento de rochas; mineração de ouro e pedras preciosas; perfuração de poços;

\footnotetext{
* Trabalho realizado na Disciplina de Pneumologia do Instituto do Coração do Hospital das Clínicas da Faculdade de Medicina da Universidade de São Paulo InCor/HC-FMUSP - São Paulo (SP) Brasil.

1. Médico Preceptor do Serviço de Pneumologia do Instituto do Coração do Hospital das Clínicas da Faculdade de Medicina da Universidade de São Paulo - InCor/ HC-FMUSP - São Paulo (SP) Brasil.

2. Médico Pós-Graduando da Disciplina de Pneumologia do Instituto do Coração do Hospital das Clínicas da Faculdade de Medicina da Universidade de São Paulo - InCor/HC-FMUSP - São Paulo (SP) Brasil.

3. Médica Assistente do Grupo de Tuberculose da Disciplina de Pneumologia do Instituto do Coração do Hospital das Clínicas da Faculdade de Medicina da Universidade de São Paulo - InCor/HC-FMUSP - São Paulo (SP) Brasil.

4. Médico Assistente do Grupo de Doenças Ocupacionais da Disciplina de Pneumologia do Instituto do Coração do Hospital das Clínicas da Faculdade de Medicina da Universidade de São Paulo - InCor/HC-FMUSP - São Paulo (SP) Brasil.

5. Professor Associado da Disciplina de Pneumologia do Instituto do Coração do Hospital das Clínicas da Faculdade de Medicina da Universidade de São Paulo InCor/HC-FMUSP - São Paulo (SP) Brasil.

Endereço para correspondência: Carlos Eduardo Galvão Barboza. Rua Luís Góis, 1313, apto. 51, Saúde, CEP 04043-350, São Paulo, SP, Brasil.

Tel 5511 3069-7202. E-mail: cebarboza@hotmail.com

Apoio financeiro: Nenhum.
}

Recebido para publicação em 9/2/2008. Aprovado, após revisão, em 3/3/2008. 
jateamento de areia; indústria de cerâmica e vidro; e fundição de ferro. ${ }^{(1,2)}$ Clinicamente, a silicose pode apresentar-se de três formas distintas: aguda, acelerada ou crônica. A forma aguda deve-se a grandes exposições à sílica e manifesta-se habitualmente em até 2 anos da exposição inicial. Na forma acelerada, os sintomas aparecem entre 2 e 10 anos. A forma crônica desenvolve-se mais de 10 anos após a exposição e costuma ser oligossintomática; pode, no entanto, evoluir com dispnéia aos esforços, de caráter progressivo. Os pacientes que apresentam a forma crônica da doença podem ter sua progressão acelerada, com evolução para óbito em poucos meses ou anos. Do ponto de vista histopatológico, a silicose caracteriza-se pela presença de granulomas, com núcleo de colágeno circundado por células epitelióides, dando origem aos nódulos silicóticos; estes se distribuem difusamente pelos pulmões e, com a progressão da doença, podem coalescer e formar grandes massas com distorção do parênquima. ${ }^{(3)}$

Além de sua importância como doença ocupacional, a silicose-ou mesmo a exposição à sílica sem doença estabelecida-associa-se a risco aumentado de desenvolvimento de diversas co-morbidades, tanto pulmonares quanto sistêmicas. Tem sido documentada, entre silicóticos, prevalências mais elevadas de doença pulmonar obstrutiva crônica, câncer de pulmão, tuberculose, micobacterioses nãotuberculosas, glomerulonefrite, artrite reumatóide, esclerodermia e outras doenças auto-imunes. ${ }^{(1,4)}$

Até o momento, não existe tratamento específico para a silicose que seja eficaz e fundamentado em ensaios clínicos. Pesquisas em países orientais mostraram melhora da função pulmonar e retardo na progressão da doença com o uso da tetrandrina, ${ }^{(5)}$ um alcalóide derivado da planta Stephania tetrandra com propriedades antioxidantes, antifibrogênicas, antiinflamatórias e imunomoduladoras.(6) Também foram testados corticosteróides e lavado broncoalveolar (LBA) terapêutico, ambos com resultados pouco promissores. ${ }^{(2)}$ Assim sendo, a conduta adequada é o afastamento da exposição. Além disso, os envolvidos no cuidado com o paciente silicótico devem estar atentos para prevenir e detectar precocemente as complicações associadas.

Este texto enfoca a associação entre silicose e o desenvolvimento de tuberculose, assim como as avaliações e as medidas preventivas apropriadas.

\section{Aspectos epidemiológicos}

A associação entre silicose e tuberculose tem sido estudada desde o início do século passado. ${ }^{(7)}$ 0 risco de portadores da silicose em desenvolver tuberculose pulmonar-comparados a controles sadios-variou entre 2,8 e 39 vezes..$^{(4,7-10)} 0$ risco para o desenvolvimento de tuberculose extrapulmonar em silicóticos também é aumentado, chegando a 3,7 vezes. ${ }^{(9,11)}$ As formas mais comuns são a pleuralresponsável por $61 \%$ dos casos $^{(11)}$-a pericárdica e a linfonodal. ${ }^{(9)}$ Quanto à relação entre as micobacterioses e as diferentes formas da silicose, estudos da literatura internacional observaram uma incidência maior nas formas aguda e acelerada. ${ }^{(12)}$ Em nosso meio, relatou-se recentemente uma prevalência de tuberculose pulmonar de 52\% em pacientes com silicose em sua forma de fibrose maciça progressiva. A maioria destes casos foi diagnosticada por baciloscopia ou cultura do escarro. ${ }^{(13)}$

Em um estudo prospectivo que avaliou 1.153 mineradores de ouro, observou-se uma incidência anual de tuberculose em silicóticos de 2,7\%, contra $0,98 \%$ nos trabalhadores sadios. Esta incidência foi proporcional à gravidade da silicose, chegando a 6,3\% nos pacientes com maior profusão de nódulos à radiografia do tórax. ${ }^{(9)}$ Um segundo estudo, no qual se avaliou a eficácia da quimioprofilaxia em pacientes com silicose (e, portanto, sem controles sadios), foi relatada uma incidência de tuberculose de 7\% ao ano no grupo que recebeu placebo. ${ }^{(11)}$

Outro estudo prospectivo, no qual foram acompanhados mais de 2.000 mineradores de ouro por 27 anos, mostrou que o risco de desenvolvimento de tuberculose pulmonar é proporcional à gravidade da silicose e à intensidade da exposição. Os trabaIhadores com maior exposição acumulada à poeira tiveram risco de apresentar tuberculose 3,22 vezes maior do que aqueles com a menor carga. Esse mesmo estudo observou intervalo médio de 6,8 anos entre o diagnóstico de silicose e o surgimento de tuberculose. ${ }^{(7)}$

Quatro estudos avaliaram a ocorrência da tuberculose em mineradores expostos à sílica, mas sem silicose. 0 risco variou entre 1,1 e 4,0 vezes em relação aos controles. ${ }^{(4,7,8,10)}$ Desses, em três estudos relacionou-se o desenvolvimento da tuberculose diretamente à exposição acumulada, de maneira semelhante a um efeito dose-resposta. ${ }^{(4,7,10)}$ A 
duração do trabalho em mineração constituiu um fator de risco adicional em um dos estudos, ${ }^{(8)}$ mas não em outro. ${ }^{(10)}$

No maior desses estudos, ${ }^{(4)}$ os autores revisaram mais de 4 milhões de certidões de óbito emitidas entre 1982 e 1995 nos Estados Unidos. Para cada caso de doença potencialmente relacionada à sílica foram pareados 5 controles. As certidões contavam com registro da ocupação, a partir da qual se atribuiu um grau de exposição à sílica, não sendo contudo possível determinar a duração da mesma. Entre os trabalhadores classificados como tendo exposição muito alta encontravam-se mineradores e operários de fundição. Foram identificados 6.570 casos de tuberculose pulmonar, 22\% dos quais apresentavam exposição à sílica. 0 odds ratio (OR) encontrado foi de 1,47 , sendo proporcional ao grau de exposição e alcançando 2,48 no grupo em que esta foi mais intensa

Por outro lado, em um segundo trabalho, foi realizado um cálculo da exposição acumulada à poeira e à sílica para cada trabalhador, a partir de medidas ambientais e história ocupacional. ${ }^{(10)}$ Os autores avaliaram transversalmente 520 mineradores de ouro quanto à presença de tuberculose pulmonar por radiografia de tórax ou história prévia. Quando se excluíram os portadores de silicose, conforme classificação radiográfica, observou-se que o risco de tuberculose foi diretamente proporcional à exposição acumulada. A prevalência variou de $20 \%$, naqueles com menor exposição, a aproximadamente $35 \%$, nos expostos à maior carga. 0 tempo de serviço isoladamente não apresentou relação independente com a prevalência de tuberculose.

Outros autores seguiram 2.255 mineradores de ouro durante 27 anos, ${ }^{(7)}$ e em 115 casos foi diagnosticada tuberculose pulmonar por bacterioscopia do escarro ou achado histológico em necropsia. De forma semelhante ao outro estudo, ${ }^{(4)}$ o risco foi proporcional à exposição cumulativa, que nesta coorte foi estimada a partir dos registros da carga horária de cada trabalhador, podendo chegar a 4,01 nos mais expostos. 0 diagnóstico da tuberculose ocorreu em média 7,6 anos após o fim da exposição, o que chama a atenção para o fato de que, mesmo após o afastamento, independentemente da presença da silicose, esta população ainda encontra-se em risco.

No último dos quatro estudos, foram avaliados 381 mineradores de ouro com cultura de escarro positiva para Mycobacterium tuberculosis e seus controles. ${ }^{(8)} 0$ risco de tuberculose foi aumentado para aqueles com tempo de trabalho em mineração superior a 10 anos, com OR de 1,9. Para exposições superiores a 15 anos, o risco foi próximo a 4 vezes o dos controles. A ocupação considerada de alta exposição à poeira (por exemplo, trabalho no subterrâneo vs. na superfície) no momento do diagnóstico mostrou tendência não-significativa de risco para a tuberculose (OR $=1,3$; intervalo de confiança: 0,82-1,94).

A ocorrência de doença por outras espécies do gênero Mycobacterium também foi estudada. Um dos estudos comparou mineradores com micobacterioses não-tuberculosas e controles sem doença pulmonar. ${ }^{(8)}$ Os seguintes fatores de risco estiveram associados ao desenvolvimento de micobacterioses: silicose $(\mathrm{OR}=5,0)$; tempo de trabalho em mineração superior a 10 anos $(\mathrm{OR}=2,6)$; tempo de trabalho em mineração superior a 20 anos $(O R=7,1)$; infecção por HIV (OR $=3,6)$; e antecedente de tuberculose $(O R=9,6)$. Em outro estudo, foram incluídos apenas mineradores com cultura de escarro positiva para micobactérias, comparando-se os infectados por $M$. tuberculosis com os portadores das demais espécies. Os fatores de risco para micobacteriose nãotuberculosa foram silicose e tratamento prévio para tuberculose (OR $=12,6$ e 3,61 , respectivamente). ${ }^{(14)}$ Em ambos os estudos, a espécie mais prevalente foi M. kansasii, responsável por aproximadamente 67\% dos casos. Em nosso serviço, temos observado uma crescente presença de $M$. kansasii em pacientes com silicose (dados não publicados).

No Brasil, um estudo investigou os fatores de risco para tuberculose na cidade de Pelotas (RS). Todos os casos diagnosticados entre 1994 e 1995 foram pareados com controles na população geral. 0 trabalho em pedreiras mostrou um risco aumentado em 4,7 vezes, enquanto que residir a uma distância inferior a $2 \mathrm{~km}$ de uma pedreira não representou risco estatisticamente significativo. Não foram avaliadas a intensidade de exposição à poeira ou a presença (e gravidade) de silicose. ${ }^{(15)}$

\section{Fisiopatologia da tuberculose na silicose}

Sabe-se pouco sobre os mecanismos pelos quais pacientes silicóticos ou expostos à sílica apresentam risco aumentado de desenvolvimento de tuberculose pulmonar e extrapulmonar. Evidências de 
estudos experimentais sugerem que a sílica modifica a resposta imune celular dos pulmões, prejudica o metabolismo e a função dos macrófagos pulmonares e, com exposição intensa, provoca a morte destas células. ${ }^{(7,11)}$ Esses achados são compatíveis com as observações de que indivíduos expostos à poeira, mesmo sem silicose estabelecida, apresentam maior incidência de tuberculose.

Outro elemento envolvido é a proteína $A$ do surfactante, que se apresenta em níveis elevados no LBA de silicóticos. 0 excesso dessa proteína parece estar associado com maior susceptibilidade à tuberculose, possivelmente por permitir a entrada das micobactérias nos macrófagos alveolares sem desencadear citotoxicidade e por inibir a formação de espécies reativas de nitrogênio pelos macrófagos ativados. ${ }^{(16,17)}$

Acredita-se ainda que os bacilos possam permanecer encapsulados no interior de nódulos silicóticos, o que seria responsável pela reativação da tuberculose nestes pacientes. ${ }^{(7)}$

\section{Aspectos diagnósticos}

\section{Silicose}

0 diagnóstico da silicose é definido a partir de uma história de exposição à sílica associada a um quadro clínico-radiológico compatível. Habitualmente, a radiografia simples de tórax é suficiente para este fim. ${ }^{(2,18)} 0$ achado radiológico característico é a presença de múltiplos nódulos menores que $10 \mathrm{~mm}$, distribuídos difusamente, mas com predomínio nas regiões superiores e posteriores dos pulmões. Os nódulos podem coalescer e formar opacidades maiores que $10 \mathrm{~mm}$, o que define a forma de fibrose maciça progressiva. 0 tipo (regular ou irregular), o tamanho (até 1,5 mm; entre 1,5 e $3 \mathrm{~mm}$; ou $>3 \mathrm{~mm}$ ) e a profusão destes nódulos formam a base para a classificação estabelecida pela Organização Internacional do Trabalho. ${ }^{(19)}$ São comuns as linfadenomegalias hilares e mediastinais, assim como sua calcificação, que por vezes assume o aspecto em "casca de ovo", bastante sugestivo do diagnóstico. Essas alterações estão presentes nas formas acelerada e crônica. A forma aguda, por sua vez, caracteriza-se por opacidades alveolares perihilares e em vidro fosco, que lembram a proteinose alveolar, dando origem ao termo silicoproteinose. ${ }^{(18)}$
A tomografia computadorizada (TC) de alta resolução do tórax fica reservada aos casos de dúvida quanto ao quadro clínico ou radiológico, a despeito de sua maior sensibilidade. ${ }^{(20)}$ Os principais achados são os nódulos difusos (de predomínio posterior e superior), opacidades centrolobulares ramificadas, nódulos subpleurais e linfadenomegalias (sendo que o padrão em "casca de ovo" esteve presente em apenas 10\% destas). As opacidades ramificadas, que representam fibrose peribronquiolar, constituem-se em sinais precoces da silicose. ${ }^{(20)}$ Já os nódulos subpleurais, ou lesões em "cera de vela", correspondem ao espessamento da pleura visceral e podem ser confundidos com as placas pleurais relacionadas ao asbesto, daí a denominação pseudoplaca. ${ }^{(21)}$ Alterações pleurais foram observadas também na silicose, sendo as principais o derrame pleural (em 12\% dos pacientes avaliados), espessamento pleural (58\% dos casos) e invaginação pleural. Esses achados foram mais prevalentes nas formas avançadas da doença, inclusive com a presença de atelectasia redonda em alguns casos de fibrose maciça progressiva. ${ }^{(22)}$

\section{Tuberculose pulmonar associada à silicose}

Nos doentes portadores de silicose, é muito importante que se exclua a coexistência de tuberculose ativa, situação em que estaria indicado o tratamento, e não a quimioprofilaxia. ${ }^{(23)}$ No entanto, o diagnóstico de tuberculose ativa sobreposta à silicose pode ser bastante difícil, particularmente em quadros iniciais, quando as manifestações clínicas podem ser frustras e as alterações radiológicas indistinguíveis das conseqüentes à silicose preexistente. ${ }^{(24)}$ Assim, diante da suspeita clínica de tuberculose ativa concomitante, deve-se proceder à investigação complementar adequada para o correto manejo do quadro.

Como avaliação complementar inicial, recomenda-se realização de baciloscopia e cultura de escarro (induzido, se necessário, pois tem boa sensibilidade) $^{(25)}$ e radiografia de tórax ${ }^{(23,26)}$ (Figura 1). Em caso de persistência de dúvidas quanto à presença de tuberculose ativa, pode-se lançar mão de broncoscopia com LBA, sempre que possível associado à biópsia transbrônquica; a realização de biópsias aumenta significativamente 0 rendimento diagnóstico do exame, mesmo nos doentes com 


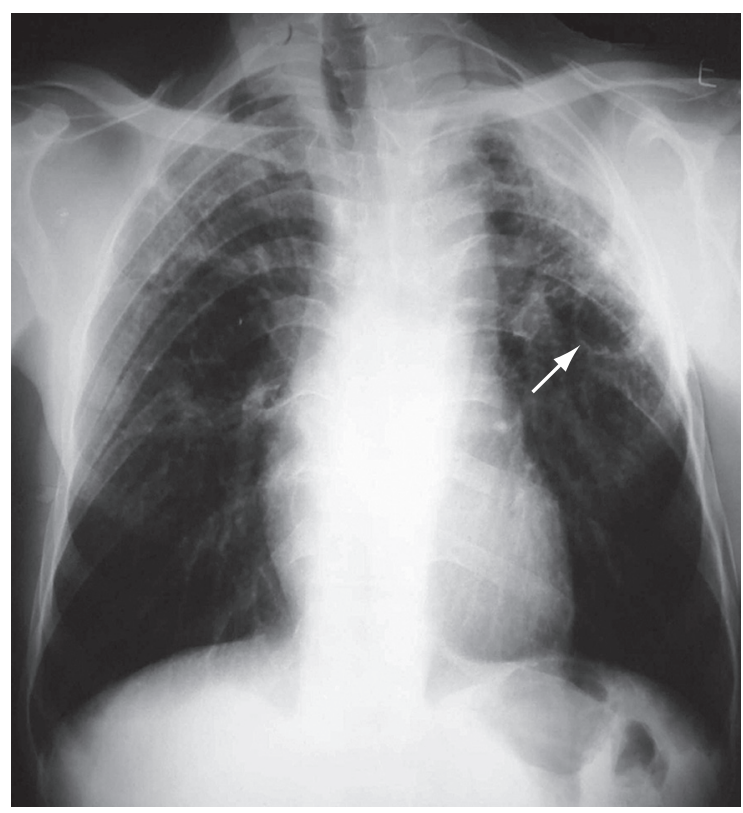

Figura 1 - Radiografia do tórax de paciente portador de silicose e tuberculose pulmonar. 0 paciente era perfurador de poços. Observa-se cavidade de paredes espessas no campo pulmonar médio à esquerda (seta).

pesquisa negativa para micobactérias no escarro e no LBA. ${ }^{(27)}$

Têm sido reconhecidos também padrões sugestivos de silicotuberculose na TC de tórax. Os principais achados compatíveis com tuberculose ativa sobreposta à silicose são cavitações de paredes espessadas, consolidações, imagens com aspecto de árvore em brotamento, assimetria das imagens nodulares e progressão rápida da doença ${ }^{(26,28,29)}$ (Figura 2).

A tomografia por emissão de pósitrons, combinada ou não à TC, estuda o metabolismo tecidual, através da captação de glicose marcada (flúor-18 fluorodesoxiglicose), e sabidamente identifica neoplasias malignas e algumas lesões benignas, como as secundárias à tuberculose. ${ }^{(30)}$ Por outro lado, tanto as lesões pulmonares quanto as linfadenomegalias conseqüentes à silicose também mostram metabolismo acelerado à tomografia por emissão de pósitrons (particularmente na forma de fibrose maciça). ${ }^{(31,32)}$ Ocorre, então, que ambas as doenças cursam com hipercaptação do radiofármaco, o que limita a correta diferenciação das lesões quanto à etiologia através desse método. Novos radiofármacos estão sendo estudados, possi- velmente com maior especificidade para as lesões induzidas pela sílica, mas ainda carecem de validação para ganharem aplicabilidade clínica. ${ }^{(33)}$

\section{Quimioprofilaxia: eficácia e segurança}

Nos últimos vinte anos, vários estudos avaliaram a utilização de quimioprofilaxia contra tuberculose em pacientes infectados pelo HIV e estabeleceram sua eficácia. Em imunocompetentes, a proteção foi evidenciada por estudos envolvendo indivíduos com seqüela pulmonar de tuberculose e contatos de pacientes bacilíferos. ${ }^{(34)}$ Poucos autores, porém, estudaram a prevenção da tuberculose em expostos à sílica, com ou sem silicose.

Um ensaio randomizado, duplo-cego, placebocontrolado avaliou o efeito de três esquemas de quimioprofilaxia em 652 silicóticos sem tuberculose ativa ou tratada. ${ }^{(11)}$ A reatividade à prova tuberculínica não constituiu um critério de inclusão. Entretanto, 94\% apresentavam reação (induração) $>10 \mathrm{~mm}$ ao início do estudo. Os indivíduos foram randomizados para receberem, de forma não supervisionada, um dos seguintes esquemas: isoniazida, $300 \mathrm{mg} / \mathrm{dia}$ por 24 semanas; isoniazida, $300 \mathrm{mg} /$ dia e rifampicina, $600 \mathrm{mg} /$ dia por 12 semanas; rifampicina, $600 \mathrm{mg} /$ dia por 12 semanas; ou placebo por 24 semanas. Ao final de 5 anos, o uso de quimioprofilaxia reduziu aproximadamente à metade o risco de desenvolvimento de tuberculose. A proporção de pacientes com tuberculose ativa no grupo placebo foi de $27 \%$, contra $13 \%$ nos que utilizaram a quimioprofilaxia (resultado combinado dos três grupos; $p<0,01)$. A incidência anual foi de $7 \%$ no grupo placebo e $4 \%$ nos grupos com uso de profilaxia. Mesmo quando se incluíram os indivíduos que não aderiram ao esquema proposto (análise por intenção de tratar), a diferença a favor da quimioprofilaxia ainda se manteve (proporção de pacientes com tuberculose, $27 \%$ vs. 17\%; $p<0,05)$. Não houve diferença significativa na eficácia dos três esquemas de quimioprofilaxia.

Neste mesmo ensaio, a suspensão do tratamento por reação adversa aconteceu em 4\% dos indivíduos randomizados para receber quimioprofilaxia e em $2 \%$ daqueles no grupo placebo. Ocorreram dois casos de hepatite sintomática, um no grupo isoniazida e outro no grupo isoniazida e rifampicina. A elevação isolada do nível sérico de alanina aminotransferase (ALT) ocorreu em até 30\% dos 


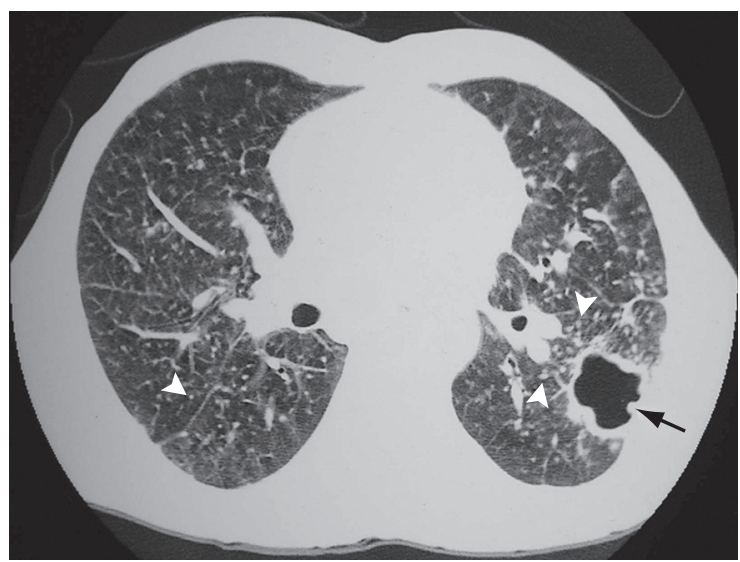

Figura 2 - Tomografia do tórax do mesmo paciente. Observar a cavidade de paredes espessas e superfície irregular no lobo inferior esquerdo (seta preta) e múltiplos nódulos distribuídos difusamente, predominantes no pulmão esquerdo (pontas de setas brancas).

que utilizaram isoniazida, porém com retorno aos valores basais após a suspensão do medicamento. Entre os que receberam apenas rifampicina, não houve diferença em relação ao placebo quanto aos níveis de ALT. Não se observou indução de cepas resistentes com o uso dos esquemas de quimioprofilaxia. ${ }^{(11)}$

A segurança da quimioprofilaxia foi avaliada em um estudo com 77 pacientes silicóticos, sem antecedentes de tuberculose e com induração na prova tuberculínica $\geq 10 \mathrm{~mm}^{\left({ }^{35)}\right.}$ Os indivíduos foram alocados para receber rifampicina, $600 \mathrm{mg} / \mathrm{dia}$ e pirazinamida, $1.500 \mathrm{mg} /$ dia por dois meses, ou isoniazida, $300 \mathrm{mg} /$ dia por seis meses. A incidência de hepatotoxicidade (definida no estudo por elevação de ALT acima de 1,5 vezes o limite superior do normal) foi maior no grupo que recebeu rifampicina e pirazinamida do que no grupo que recebeu isoniazida apenas (47,5\% vs. 13,9\%; $p<0,01)$. Quando se delimitou a análise a aumentos superiores a 5 vezes, os resultados foram, respectivamente, $35 \%$ e $2,8 \%$ ( $p<0,001)$. A ocorrência de sintomas sugestivos de hepatite foi, respectivamente, de $15 \%$ e 2,7\%; porém, a exclusão de hepatite por vírus foi feita em apenas 4 dos 7 pacientes em questão. De forma semelhante, o tratamento foi interrompido devido à toxicidade hepática em maior número de indivíduos do grupo rifampicina e pirazinamida (35\% vs. 5,6\%; $p<0,01)$.

A aderência a esquemas prolongados e potencialmente tóxicos representa mais uma dificuldade da quimioprofilaxia. No grupo alocado para receber rifampicina e pirazinamida por dois meses, a aderência foi de 55\%. Entre aqueles randomizados para uso de isoniazida por seis meses, 63,9\% completaram o esquema. A diferença de aderência entre os grupos não se mostrou estatisticamente significativa. ${ }^{(35)}$

\section{Recomendações das diretrizes}

A associação entre exposição à sílica, com ou sem silicose, e o risco de desenvolvimento de tuberculose e outras micobacterioses está bem documentada na literatura. 0 uso de quimioprofilaxia nesta população, por outro lado, não conta com evidências igualmente extensas. Apesar de existirem poucos estudos avaliando criteriosamente a indicação, eficácia, segurança e duração para os diferentes esquemas, tanto as diretrizes nacionais quanto as internacionais recomendam quimioprofilaxia para os portadores da silicose.

0 II Consenso Brasileiro de Tuberculose recomenda que pacientes com silicose e que sejam reatores fortes à prova tuberculínica (induração $\geq 10 \mathrm{~mm}$ ) recebam isoniazida, na dose de $300 \mathrm{mg} / \mathrm{dia}$ (ou $10 \mathrm{mg} / \mathrm{kg} / \mathrm{dia}$ ) durante seis meses. Considerando-se que a quimioprofilaxia por períodos maiores tem eficácia semelhante, deve-se reservar o esquema de nove meses para grupos de muito alto risco para tuberculose. ${ }^{(23)}$

A American Thoracic Society, ${ }^{(34)}$ da mesma forma, recomenda a realização da prova tuberculínica em portadores de silicose. No caso de ser positiva (induração $\geq 10 \mathrm{~mm}$ )-e após a exclusão de tuberculose ativa-recomenda a instituição de profilaxia, com um dos quatro possiveis esquemas: isoniazida por nove meses; isoniazida por seis meses; rifampicina e pirazinamida por dois a três meses; ou rifampicina por quatro meses. Embora não haja uma recomendação específica para silicóticos, o esquema preconizado por essa sociedade é de isoniazida por nove meses, independente da situação sorológica para HIV. A dosagem de bilirrubinas e enzimas hepáticas na avaliação inicial é indicada apenas para os indivíduos considerados de risco elevado para hepatopatia, como etilistas, gestantes e portadores do HIV. ${ }^{(34)}$

Nossa opinião é a de que pacientes com silicose e também aqueles com exposição à sílica há mais de 10 anos, mesmo sem a doença, sejam submetidos à 
prova tuberculínica na avaliação inicial, visto que esta população tem risco de adoecimento reconhecidamente aumentado. ${ }^{(8)}$ Caso resulte em não-reator, recomenda-se repeti-la em duas semanas para confirmação, considerando que a exposição à prova tuberculínica pode estimular a memória imunológica contra o bacilo. Persistindo o resultado negativo, o exame deve ser realizado anualmente, ${ }^{(36)}$ por tempo indeterminado. Se positivo (induração $\geq 10 \mathrm{~mm}$ ), deve-se instituir quimioprofilaxia com isoniazida na dose de $300 \mathrm{mg} / \mathrm{dia}$ (ou $10 \mathrm{mg} / \mathrm{kg} / \mathrm{dia}$ ) por seis meses. Embora careça de comprovação quanto ao seu benefício, esta posição é compartilhada por especialistas na literatura internacional. ${ }^{(37)}$

\section{Considerações finais}

A silicose é uma doença prevalente e atualmente sem tratamento específico. Seu manejo deve centrar-se em afastar o trabalhador da exposição e em evitar agravamentos, como por exemplo, garantindo a vacinação contra influenza e pneumococos. Entre as potenciais complicações, destaca-se a tuberculose, para a qual os silicóticos podem apresentar um risco até 40 vezes maior do que o da população em geral. A eficácia da quimioprofilaxia já está bem estabelecida para os portadores do HIV e, em menor grau, para os portadores de silicose. Portanto, no seguimento destes pacientes, deve-se avaliar o risco individual para o desenvolvimento da tuberculose por meio da prova tuberculínica e, para os reatores fortes (induração $\geq 10 \mathrm{~mm}$ ), indicar a quimioprofilaxia. Mais estudos, entretanto, são necessários para consolidar esta indicação, bem como identificar o melhor esquema quanto à medicação de escolha, dose e duração.

Em relação aos expostos à sílica que não apresentam a doença, população também de risco para a tuberculose, seria recomendável a instituição da quimioprofilaxia naqueles com exposição superior a 10 anos e reação forte à prova tuberculínica. Entretanto, protocolos de estudo precisam ainda ser desenvolvidos para validar esta conduta.

\section{Referências}

1. Rice FL. Crystalline silica, quartz. Concise international chemical assessment document, 24. Geneva: World Health Organization; 2000.

2. Terra-Filho M, Santos UP. Silicosis. J Bras Pneumol. 2006;32(Suppl 2):S41-7.
3. Castranova V, Vallyathan V. Silicosis and coal workers' pneumoconiosis. Environ Health Perspect. 2000;108(Suppl 4):675-84.

4. Calvert GM, Rice FL, Boiano JM, Sheehy JW, Sanderson WT. Occupational silica exposure and risk of various diseases: an analysis using death certificates from 27 states of the United States. Occup Environ Med. 2003;60(2):122-9.

5. Xie QM, Tang HF, Chen JQ, Bian RL. Pharmacological actions of tetrandrine in inflammatory pulmonary diseases. Acta Pharmacol Sin. 2002;23(12):1107-13.

6. Lai JH. Immunomodulatory effects and mechanisms of plant alkaloid tetrandrine in autoimmune diseases. Acta Pharmacol Sin. 2002;23(12):1093-101.

7. Hnizdo E, Murray J. Risk of pulmonary tuberculosis relative to silicosis and exposure to silica dust in South African gold miners. Occup Environ Med. 1998;55(7):496-502. Erratum in: Occup Environ Med 1999;56(3):215-6.

8. Corbett EL, Churchyard GJ, Clayton T, Herselman P, Williams B, Hayes R, et al. Risk factors for pulmonary mycobacterial disease in South African gold miners. A case-control study. Am J Respir Crit Care Med. 1999;159(1):94-9.

9. Cowie RL. The epidemiology of tuberculosis in gold miners with silicosis. Am J Respir Crit Care Med. 1994;150(5 Pt 1):1460-2.

10. teWaternaude JM, Ehrlich Rl, Churchyard GJ, Pemba $\mathrm{L}$, Dekker K, Vermeis M, et al. Tuberculosis and silica exposure in South African gold miners. Occup Environ Med. 2006;63(3):187-92.

11. A double-blind placebo-controlled clinical trial of three antituberculosis chemoprophylaxis regimens in patients with silicosis in Hong Kong. Hong Kong Chest Service/Tuberculosis Research Centre, Madras/British Medical Research Council. Am Rev Respir Dis. 1992;145(1):36-41.

12. Adverse effects of crystalline silica exposure. American Thoracic Society Committee of the Scientific Assembly on Environmental and Occupational Health. Am J Respir Crit Care Med. 1997;155(2):761-8.

13. Ferreira AS, Moreira VB, Ricardo HM, Coutinho R, Gabetto JM, Marchiori E. Progressive massive fibrosis in silica-exposed workers. High-resolution computed tomography findings. J Bras Pneumol. 2006; 32(6):523-8.

14. Sonnenberg P, Murray J, Glynn JR, Thomas RG, GodfreyFaussett P, Shearer S. Risk factors for pulmonary disease due to culture-positive M. tuberculosis or nontuberculous mycobacteria in South African gold miners. Eur Respir J. 2000;15(2):291-6.

15. Menezes AMB, Costa JD, Gonçalves H, Saul M, Menezes M, Soila L et al. Incidência e Fatores de Risco para Tuberculose em Pelotas, Uma Cidade do Sul do Brasil. Rev Bras Epidemiol. 1998;1(1):50-60.

16. Pasula R, Wright JR, Kachel DL, Martin WJ 2nd. Surfactant protein A suppresses reactive nitrogen intermediates by alveolar macrophages in response to Mycobacterium tuberculosis. J Clin Invest. 1999;103(4):483-90.

17. Gold JA, Hoshino Y, Tanaka N, Rom WN, Raju B, Condos $\mathrm{R}$, et al. Surfactant protein A modulates the inflammatory response in macrophages during tuberculosis. Infect Immun. 2004;72(2):645-50.

18. Davis GS. Silicosis. In: Hendick DJ, Burge PS, Beckett WS, Churg A, editors. Occupational disorders of the lung: recognition, management and prevention. London: W. B. Saunders; 2002. p. 105-127. 
19. Organização Internacional do Trabalho. Diretrizes para Utilização da Classificação Internacional da 01T de Radiografias de Pneumoconioses. São Paulo: 01T; 2005.

20. Antao VC, Pinheiro GA, Terra-Filho M, Kavakama J, Müller NL. High-resolution CT in silicosis: correlation with radiographic findings and functional impairment. J Comput Assist Tomogr. 2005;29(3):350-6.

21. Müller NL, Fraser RS, Colman NC, Paré PD. Doença Pulmonar Causada por Inalação de ou Aspiração de Partículas, de Sólidos ou de Líquidos. In: Muller NL, editors. Diagnóstico radiológico das doenças do tórax. Rio de Janeiro: Guanabara Koogan; 2003. p. 511-553.

22. Arakawa H, Honma K, Saito Y, Shida H, Morikubo H, Suganuma $\mathrm{N}$, et al. Pleural disease in silicosis: pleural thickening, effusion, and invagination. Radiology. 2005;236(2):685-93.

23. Sociedade Brasileira de Pneumologia e Tisiologia. Il Consenso Brasileiro de Tuberculose: Diretrizes Brasileiras para Tuberculose 2004. J Pneumol. 2004;30(Supl 1):S1-86.

24. Snider DE Jr. The relationship between tuberculosis and silicosis. Am Rev Respir Dis. 1978;118(3):455-60

25. Conde MB, Soares SL, Mello FC, Rezende VM, Almeida LL, Reingold $\mathrm{AL}$, et al. Comparison of sputum induction with fiberoptic bronchoscopy in the diagnosis of tuberculosis: experience at an acquired immune deficiency syndrome reference center in Rio de Janeiro, Brazil. Am J Respir Crit Care Med. 2000;162(6):2238-40.

26. Bombarda S, Figueiredo CM, Funari MB, Soares Jr J, Seiscento M, Terra-Filho M. Imagem em tuberculose pulmonar. J Pneumol. 2001;27(6):329-40.

27. Charoenratanakul S, Dejsomritrutai W, Chaiprasert A. Diagnostic role of fiberoptic bronchoscopy in suspected smear negative pulmonary tuberculosis. Respir Med. 1995;89(9):621-3.
28. Lee KS, Hwang JW, Chung MP, Kim H, Kwon 0J. Utility of CT in the evaluation of pulmonary tuberculosis in patients without AIDS. Chest. 1996;110(4):977-84.

29. Chong S, Lee KS, Chung MJ, Han J, Kwon 0J, Kim TS. Pneumoconiosis: comparison of imaging and pathologic findings. Radiographics. 2006;26(1):59-77.

30. Bombarda S, Soares Jr J, Terra-Filho M. Estudo do metabolismo da glicose na tuberculose pulmonar ativa utilizando a tomografia por emissão de pósitrons (18F-FDG PET). J Pneumol. 2002;28(5):270-6.

31. Chang JM, Lee HJ, Goo JM, Lee HY, Lee JJ, Chung JK, et al. False positive and false negative FDG-PET scans in various thoracic diseases. Korean J Radiol. 2006;7(1):57-69.

32. O'Connell M, Kennedy M. Progressive massive fibrosis secondary to pulmonary silicosis appearance on F-18 fluorodeoxyglucose PET/CT. Clin Nucl Med. 2004;29(11):754-5.

33. Wallace WE, Gupta NC, Hubbs AF, Mazza SM, Bishop HA, Keane MJ, et al. Cis-4-[(18)F]fluoro-L-proline PET imaging of pulmonary fibrosis in a rabbit model. J Nucl Med. 2002;43(3):413-20.

34. American Thoracic Society. Targeted Tuberculin Testing and Treatment of Latent Tuberculosis Infection. Am J Respir Crit Care Med. 2000; 161(4): S221-S247.

35. Leung CC, Law WS, Chang KC, Tam CM, Yew WW, Chan $\mathrm{CK}$, et al. Initial experience on rifampin and pyrazinamide vs isoniazid in the treatment of latent tuberculosis infection among patients with silicosis in Hong Kong. Chest. 2003;124(6):2112-8.

36. Balaan DR, Banks DE. Silicosis. In: Rom WN, editor. Environmental and occupational medicine. Boston: Little, Brown; 1992. p. 345-58.

37. Rees D, Murray J. Silica, silicosis and tuberculosis. Int J Tuberc lung Dis. 2007;11(5):474-84. 